\title{
In Vivo Detection of CPP-II5 Target Engagement in Human Brain
}

\author{
Andrew P Prescot ${ }^{*, 1}$, Steven R Miller ${ }^{2}$, Gary Ingenito ${ }^{2}$, Rebekah S Huber ${ }^{3}$, Douglas G Kondo ${ }^{3,4}$ and $^{-}$ \\ Perry F Renshaw ${ }^{3,4}$
}

'Department of Radiology and Imaging Sciences, University of Utah School of Medicine, Salt Lake City, UT, USA; ${ }^{2}$ Catalyst Pharmaceuticals, Coral Gables, FL, USA; ${ }^{3}$ Department of Psychiatry, University of Utah School of Medicine, Salt Lake City, UT, USA; ${ }^{4}$ Rocky Mountain Mental IIIness Research, Education, and Clinical Center (MIRECC), Department of Veterans Affairs Medical Center, Salt Lake City, UT, USA

\begin{abstract}
CPP-II5, a next-generation $\gamma$-amino butyric acid (GABA)-aminotransferase (AT) inhibitor, shows comparable pharmacokinetics, improved safety and tolerability, and a more favorable toxicity profile when compared with vigabatrin. The pharmacodynamic characteristics of CPP-I I 5 remain to be evaluated. The present study employed state-of-the-art proton magnetic resonance spectroscopy techniques to measure changes in brain GABA+ (the composite resonance of GABA, homocarnosine, and macromolecules) concentrations in healthy subjects receiving oral daily doses of CPP-I I 5 or placebo. Six healthy adult males were randomized to receive either single daily $80 \mathrm{mg}$ doses of CPP- I I $5(n=4)$ or placebo $(n=2)$ for 6 , I0, or I 4 days. Metabolite-edited spectra and two-dimensional J-resolved spectroscopy data were acquired from the parietal-occipital cortex and supplementary motor area in all subjects. Four scans were performed in each subject that included a predrug baseline measure, two scans during the dosing timeframe, and a final scan that occurred I week after drug cessation. CPP-II5 induced robust and significant increases in brain GABA+ concentrations that ranged between 52 and $141 \%$ higher than baseline values. Elevated GABA+ concentrations returned to baseline values following drug clearance. Subjects receiving placebo showed no significant changes in GABA+ concentration. CPP-1 I5-induced changes were exclusive to GABA and homocarnosine, and CPP-II 5 afforded brain GABA+ concentration changes comparable to or greater than previous vigabatrin spectroscopy studies in healthy epilepsy-naive subjects. The return to baseline GABA+ concentration indicates the reversible GABA-AT resynthesis following drug washout. These preliminary data warrant further spectroscopy studies that characterize the acute pharmacodynamic effects of CPP- I 5 with additional dose-descending measures.
\end{abstract}

Neuropsychopharmacology (2018) 43, 646-654; doi:I0.1038/npp.2017.I56; published online 6 September 2017

\section{INTRODUCTION}

The modulation of $\gamma$-amino butyric acid (GABA), the major inhibitory neurotransmitter in mammalian brain, is a major focus for the development of pharmacotherapies designed to treat a range of psychiatric illnesses, substance abuse disorders, and neurologic diseases (Yogeeswari et al, 2006; Gajcy et al, 2010). GABA-aminotransferase (GABA-AT) is responsible for GABA catabolism, and its irreversible inactivation by suicide inhibitors leads to elevated intracellular GABA concentrations (Jung et al, 1977). Vigabatrin is a commonly used GABA-AT inhibitor used to treat seizure disorders including epilepsy, complex partial seizures, and infantile spasms (Grant and Heel, 1991). Proton $\left({ }^{1} \mathrm{H}\right)$ magnetic resonance spectroscopy (MRS) studies have reported vigabatrin-induced GABA concentration levels that

*Correspondence: Dr AP Prescot, Department of Radiology and Imaging Sciences, University of Utah School of Medicine, 383 Colorow Drive, Salt Lake City, UT 84I08, USA, Tel: + 801587 |44I, Fax: + I 801585 5375, E-mail: andrew.prescot@utah.edu

Received 9 February 20 17; revised I I July 20 17; accepted I7 July 20 I7; accepted article preview online 25 July 2017 are $\sim 100$ to $200 \%$ higher than baseline levels in epilepsy patients (Mattson et al, 1994), although moderate GABA concentration increases of $\sim 60 \%$ was reported in ${ }^{1} \mathrm{H}$ MRS studies of healthy control subjects (Weber et al, 1999). However, poorly controlled epileptic subjects tend to have lower baseline GABA levels than healthy controls (Petroff et al, 1996) that could in part explain the larger GABA changes seen in epileptic patients (Mattson et al, 1994) compared with healthy subjects (Weber et al, 1999). Another ${ }^{1} \mathrm{H}$ MRS study reported the effects of vigabatrin on brain GABA, glutamate (Glu), and glutamine (Gln) levels in patient populations, identifying significantly elevated GABA and Gln concentrations, with a concomitant reduction in Glu (Petroff et al, 1995). Vigabatrin has also been shown to increase levels of brain homocarnosine, a dipeptide of GABA and histidine, that is thought to contribute to improved seizure control in epilepsy patients (Petroff et al, 1998).

High doses of vigabatrin (3-4 g per day) are required to achieve therapeutic effects, and chronic long-term treatment in humans is associated with significant adverse effects, including permanent peripheral visual field defects, intramyelinic edema (microvacuolation), and an array of central 
nervous system disturbances (Perucca and Gilliam, 2012). Vigabatrin is approved for the management of refractory complex partial seizures in adult patients who have failed to respond to other lines of anticonvulsant treatments and, for some patients, is administered as a monotherapy if the potential benefits outweigh the associated risks.

Preclinical data for (1S, 3S)-3-amino-4-difluoromethylenyl-1-cyclopentanoic acid (CPP-115), a next-generation GABA-AT inhibitor, show that significantly lower drug dosages afford comparable pharmacokinetics, improved tolerability, and a more favorable toxicity profile when compared with vigabatrin (Pan et al, 2012; Briggs et al, 2014). A single case report has also documented the efficacy of CPP-115 in a patient with infantile spasms, demonstrating improved clinical symptoms with no evidence of retinal dysfunction (Doumlele et al, 2016). The pharmacodynamic effects of CPP-115 have yet to be established, and the present study was designed to employ noninvasive state-of-the-art ${ }^{1} \mathrm{H}$ MRS procedures to measure cortical GABA+ concentration changes across multiple brain regions in healthy adult subjects receiving either oral daily doses of CPP-115 or placebo. 'GABA+' denotes the composite MRS resonance that contains co-edited free GABA, homocarnosine, and macromolecule (MM) signals. Metabolite-specific editing ${ }^{1} \mathrm{H}$ MRS methods were used to measure GABA+ levels, whereas optimized two-dimensional (2D) J-resolved ${ }^{1} \mathrm{H}$ MRS techniques (Schulte et al, 2006) were employed for measuring other MRS-detectable metabolites. Compared with conventional ${ }^{1} \mathrm{H}$ MRS approaches, 2D J-resolved ${ }^{1} \mathrm{H}$ MRS techniques have been shown to offer improved reproducibility and quantification precision, particularly for Glu and Gln measurements (Schulte and Boesiger, 2006; Prescot and Renshaw, 2013).

\section{MATERIALS AND METHODS}

\section{Human Subjects}

Clinical aspects of the present study were managed by a clinical study unit (CSU; PRA Health Sciences, Salt Lake City, UT). Imaging/spectroscopy procedures were performed at the University of Utah. Institutional review boards (IRBs) specific to each site had approved their respective protocols that met criteria for investigations in human subjects (https://www.hhs.gov/ohrp/regulations-and-policy/belmontreport/index.html last accessed on 06/07/2017).

Subjects were screened at the CSU and assessed for enrollment using the following inclusion criteria: male participants of 18-65 years of age; body mass index 19$32 \mathrm{~kg} / \mathrm{m}^{2}$; willing and able to provide written informed consent and comply with study procedures; agreed to use double-barrier contraception during the study and for 1 month afterward; normal systolic blood pressure (90$140 \mathrm{~mm} \mathrm{Hg}$ ), diastolic blood pressure (50-90 $\mathrm{mm} \mathrm{Hg})$, and resting pulse rate (40-90 beats per min (b.p.m.)); normal organ and bone marrow function at screening and predose; willing to abstain from illicit drugs, alcohol, and tobacco use during study participation.

A subject was not eligible for the study if one or more of the following criteria applied: medical history and/or findings on physical examination indicating the presence of clinically significant illness; clinically significant abnormalities of vital signs or clinical laboratory results; presence or recent history (28 days before screening) of active and clinically significant gastrointestinal, renal, cardiovascular, hepatic, metabolic, allergic, dermatologic, hematologic, pulmonary, neurological, or psychiatric illness; history of stomach or intestinal surgery or resection that would potentially alter absorption and/or excretion of orally administered drugs; clinically significant electrocardiographic abnormalities including corrected QT interval (QTc $\geqslant 450 \mathrm{~ms}$ ); contraindications to MRI scanning including the presence of metallic surgical hardware, cardiac pacemaker, stents, mechanical heart valves, artificial limbs, brain stimulator devices, implanted drug pumps, cochlear implants, ocular implants or known metal fragments in eyes, exposure to shrapnel or metal filings, magnetic dental implants, or permanent retainer; history of clinically significant claustrophobia; subject had obtained a new tattoo within 30 days before first dose; clinically significant ophthalmologic disease that would preclude safety monitoring, was undergoing treatment for ocular disease, or intended to have ocular surgery performed during the time of study participation; use of tobacco-containing or nicotine-containing products within 6 months before screening; use of any prescription, illegal, or investigational drug within 28 days before first scheduled dose; use of any over-the-counter drug, including vitamins, minerals, dietary/herbal supplements, or grapefruit or grapefruit juice within 14 days before first scheduled dose; history of recent (within 6 months) drug or alcohol abuse, as defined in DSM IV; use of alcohol, caffeine, or poppy seedcontaining foods or beverages within $72 \mathrm{~h}$ before first dose; positive urine drug screen, urine cotinine test, or alcohol breath test at screening or check-in to the CSU; positive serology for the surface antigen of hepatitis B, hepatitis $C$, or human immunodeficiency virus antibody screen; donation of blood or plasma to a blood bank or for a clinical study within 28 days before first scheduled dose; receipt of blood products within 2 months before screening; any condition that, in the opinion of the CSU primary investigator, would render the subject unsuitable for the clinical study; known history of epilepsy or seizures.

Six healthy adult male subjects (mean age $\pm \mathrm{SD}=34.2 \pm$ 16.8 years) were enrolled into a double-blind, randomized, placebo-controlled study. Subjects received either a single oral daily $80 \mathrm{mg}$ dose (the current maximum daily dose approved by the FDA) of CPP-115 $(n=4)$ or placebo $(n=2)$ for 6,10 , or 14 continuous days, with placebo and CPP-115 administered as noncarbonated artificially sweetened beverages. The unblinded study subject information (treatment allocation/treatment duration) was as follows: subject 1: CPP-115/14 days, subject 2: placebo/14 days, subject 3: CPP-115/10 days, subject 4: CPP-115/10 days, subject 5: placebo/6 days, and subject 6: CPP-115/6 days. Figure 1

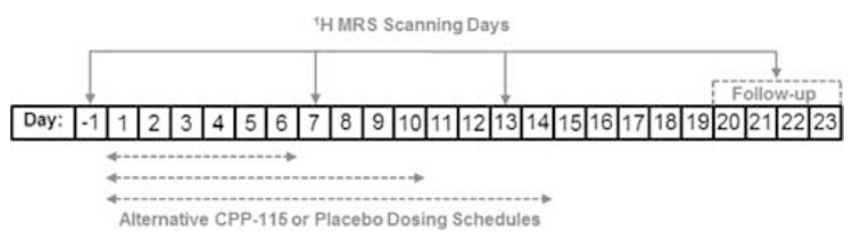

Figure I The study timeline, showing the positioning of ' $\mathrm{H}$ MRS scanning days in relation to the three possible CPP-II5 or placebo treatment schedules that were randomly assigned to subjects. 
shows the timing of four ${ }^{1} \mathrm{H}$ MRS measurements in relation to the dosing regimen. All subjects underwent a baseline ${ }^{1} \mathrm{H}$ MRS scan at day -1 with study drug or placebo dosing initiated at day 1 . Two ${ }^{1} \mathrm{H}$ MRS scans were then performed at day 7 and day 13, with the scans occurring $2.5 \mathrm{~h}$ after drug administration, and at approximately the same time of day as baseline (day -1$)$ measures. The fourth and final ${ }^{1} \mathrm{H}$ MRS measurement (follow-up) occurred within the day 20 to day 23 window, to allow for a minimum 7 -day washout period from the final dose day. Study subjects remained at the CSU facility for the first 16 study days, and returned within the day 20 to day 23 window for final clinical assessments. The CSU also was responsible for transporting study subjects to the University of Utah for imaging and MRS procedures.

\section{MRI and ${ }^{1} \mathrm{H}$ MRS Data Acquisition}

${ }^{1} \mathrm{H}$ MRI and MRS measurements were performed using a 2.89 Tesla Siemens (Erlargen, Germany) Verio whole-body MRI scanner housed at the University of Utah's University Neuropsychiatric Institute. A circularly polarized body coil was used for radiofrequency (RF) transmission with a manufacturer-supplied 12-channel phased-array receive-only head coil employed for signal reception. Subjects were positioned supine and foam pads were used to fixate the subject's head within the RF coil housing. Three orthogonal low-resolution proton-weighted gradient echo (repetition time/echo time $(\mathrm{TR} / \mathrm{TE})=20 / 5 \mathrm{~ms}$; field of view $(\mathrm{FOV})=$ $280 \times 280 \mathrm{~mm}$; matrix size $=192 \times 144 ; 8 \mathrm{~mm}$ slice thickness) localizer images were obtained to confirm optimal head positioning at the receive-only coil center. Three-dimensional (3D) high-resolution T1-weighted, magnetizationprepared, rapid gradient echo (MP-RAGE; TR/TE/TI = $2000 / 3.53 / 1100 \mathrm{~ms} ; \quad \mathrm{FOV}=256 \times 256 \times 224 \mathrm{~mm}$; isotropic $1 \mathrm{~mm}$ in-plane resolution) MR images were then obtained and used to facilitate accurate MRS voxel positioning.

The present study acquired ${ }^{1} \mathrm{H}$ MRS data from two separate single voxels measuring $25 \times 25 \times 30 \mathrm{~mm}^{3}$, positioned along the midline of the parietal-occipital cortex (POC) and the supplementary motor area (SMA). The largest voxel dimension was aligned along the superiorinferior and left-right orientations for the POC and SMA, respectively (see Figure 2). For baseline MRS scans (day - 1), the sagittal and axial MP-RAGE slices extending through the center of the MRS voxel were saved to an image file that was subsequently used to facilitate manual POC and SMA voxel repositioning at the three later MRS scans on a subjectspecific basis. Local $\mathrm{B}_{0}$ shimming for POC and SMA voxels was performed using the manufacturer-supplied FASTMAP (Gruetter, 1993) routine that resulted in water signal linewidths (LWs; unsuppressed real component) of $\leqslant 8 \mathrm{~Hz}$.

A MEGA-PRESS metabolite-specific editing ${ }^{1} \mathrm{H}$ MRS sequence (Mescher et al, 1998) was used for determining POC and SMA GABA+ concentrations. MEGA-PRESS is a variant of $J$-editing ${ }^{1} \mathrm{H}$ MRS where frequency-selective Gaussian-modulated RF pulses are applied in an interleaved manner to selectively refocus the GABA+ 3.0 p.p.m. resonance. This is achieved by applying the Gaussian refocusing pulses either at 1.9 p.p.m. ('on' resonance for the GABA C3 protons) or at 7.5 p.p.m. ('off resonance condition). Multiple 'on' and 'off resonance spectra are collected to attain a suitable signal-to-noise ratio, and the subsequent subtraction of 'off data from 'on' data affords a J-edited spectrum with a refocused GABA+ 3.0 p.p.m. signal that is free of the larger dominating creatine methyl resonance. MEGA-PRESS data were collected using the following acquisition parameters: $\mathrm{TR}=2000 \mathrm{~ms}, \mathrm{TE}=68 \mathrm{~ms}$, number of signal averages $(\mathrm{NAV})=256$ 'on' and 256 'off. Potential changes in other POC and SMA brain metabolites, including total creatine (Cre), choline compounds (Cho), $\mathrm{N}$-acetyl aspartate (NAA), Glu, Gln, and myo-inositol (Ins), were measured using a maximum echo-sampled 2D J-resolved ${ }^{1} \mathrm{H}$ MRS sequence (Schulte et al, 2006) with the following parameters: $\mathrm{TR}=2000 \mathrm{~ms}$, $\mathrm{TE}$ range $=31-229 \mathrm{~ms}, \Delta \mathrm{TE}=2 \mathrm{~ms}$, NAV per $\mathrm{TE}=4$. Total measurement time for setup, imaging, and spectroscopy did not exceed $80 \mathrm{~min}$ for each scan session.

\section{MRI and ${ }^{1} \mathrm{H}$ MRS Data Processing}

Skull stripping and whole-brain tissue-type segmentation was performed on MP-RAGE images using the Brain Extraction Tool (BET; Smith, 2002) and Fast Automated Segmentation Tool (FAST; Zhang et al, 2001), respectively, provided with the FMRIB software library (Smith et al, 2004). MATLAB (R2010b, The MathWorks, Natick, MA) functions were then used to extract the $3 \mathrm{D}$ volume corresponding to the positioned MRS voxel to obtain within-voxel gray matter (GM), white matter (WM), and cerebrospinal fluid (CSF) tissue content for each subject. The GM and WM fractional content was calculated as a percentage of total brain tissue, eg, $100 \times \mathrm{GM} /(\mathrm{GM}+\mathrm{WM})$.

GABA-edited MEGA-PRESS ${ }^{1} \mathrm{H}$ MRS data were processed and quantified using the freely available Gannet software (Edden et al, 2014) using the raw Siemens 'TWIX' data format. Gannet applies a fully automated phase and frequency correction procedure to each 'on' and 'off' signal average before averaging across the two spectral subtypes and subtracting the corrected 'off from the 'on' edited data. Gannet then employs an automated peak fitting routine to fit a Gaussian lineshape to the edited GABA+ resonance at 3.0 p.p.m. The software's default preprocessing and peak fitting parameters, including water-based signal correction and application of a $3 \mathrm{~Hz}$ exponential line broadening filter, were used for all MEGA-PRESS data. To extract GABA+ concentration, a Cre concentration of $8 \mathrm{mM}$ was assumed and applied as a multiplication factor to the Gannetoutputted GABA+/Cre ratio. The $2 \mathrm{D} J$-resolved ${ }^{1} \mathrm{H}$ MRS data were processed using the exact procedures described elsewhere (Schulte and Boesiger, 2006; Prescot and Renshaw, 2013).

MEGA-PRESS subspectra were used to quantify changes in POC and SMA homocarnosine concentrations. The 'onresonance' subspectra were used to avoid homocarnosine attenuation effects caused by the frequency-selective refocusing pulse centered at 7.5 p.p.m. in the 'off-resonance' data. After exponential line-broadening $(4 \mathrm{~Hz})$ and fast Fourier transformation, the signal integrals around 7.05 p.p.m. (homocarnosine) and 3.0 p.p.m. (creatine) were calculated. Following correction for number of proton nuclei contributing to each peak, estimates for homocarnosine concentrations also assumed a creatine concentration reference of $8 \mathrm{mM}$. 

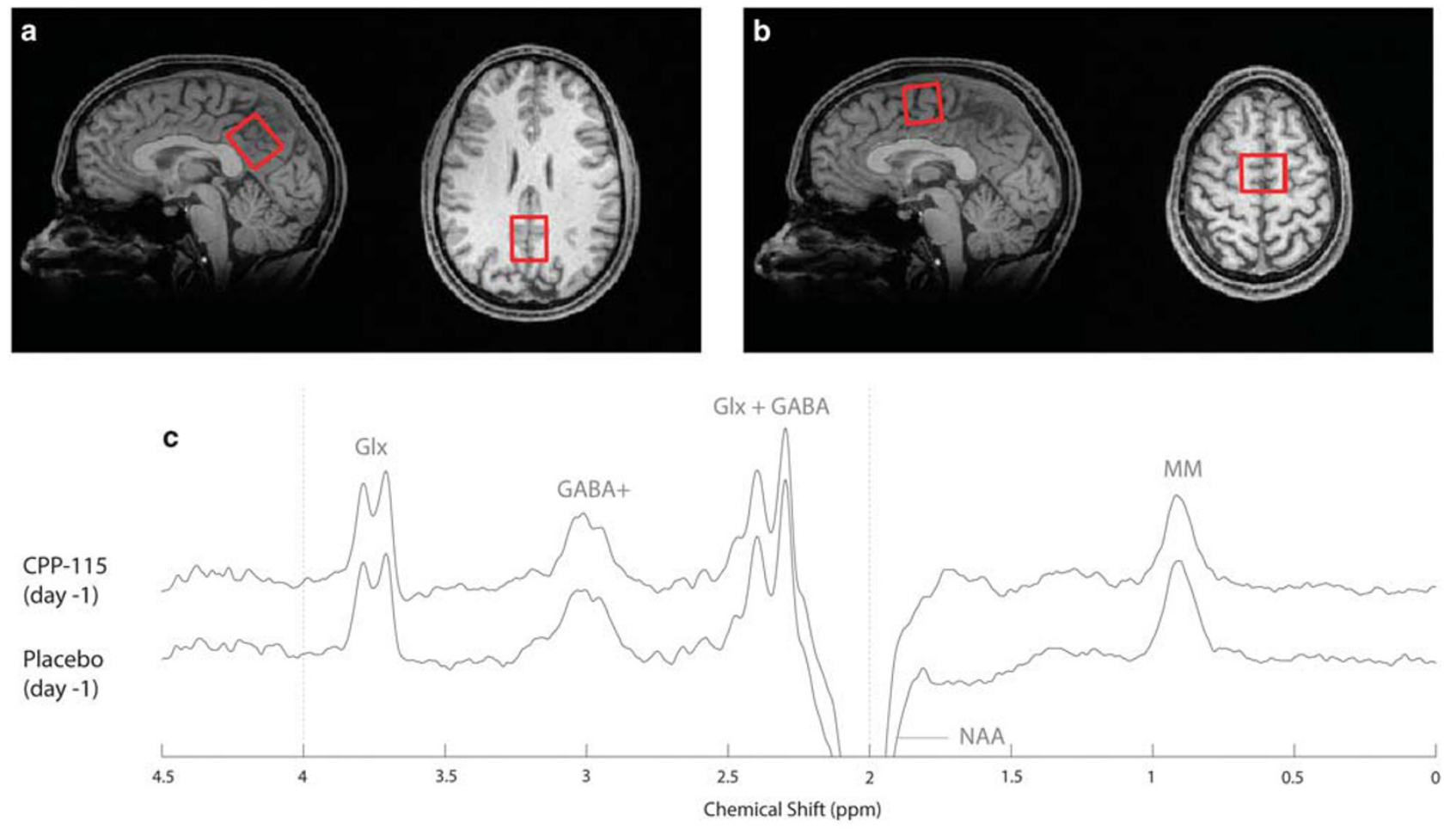

Visit Key:
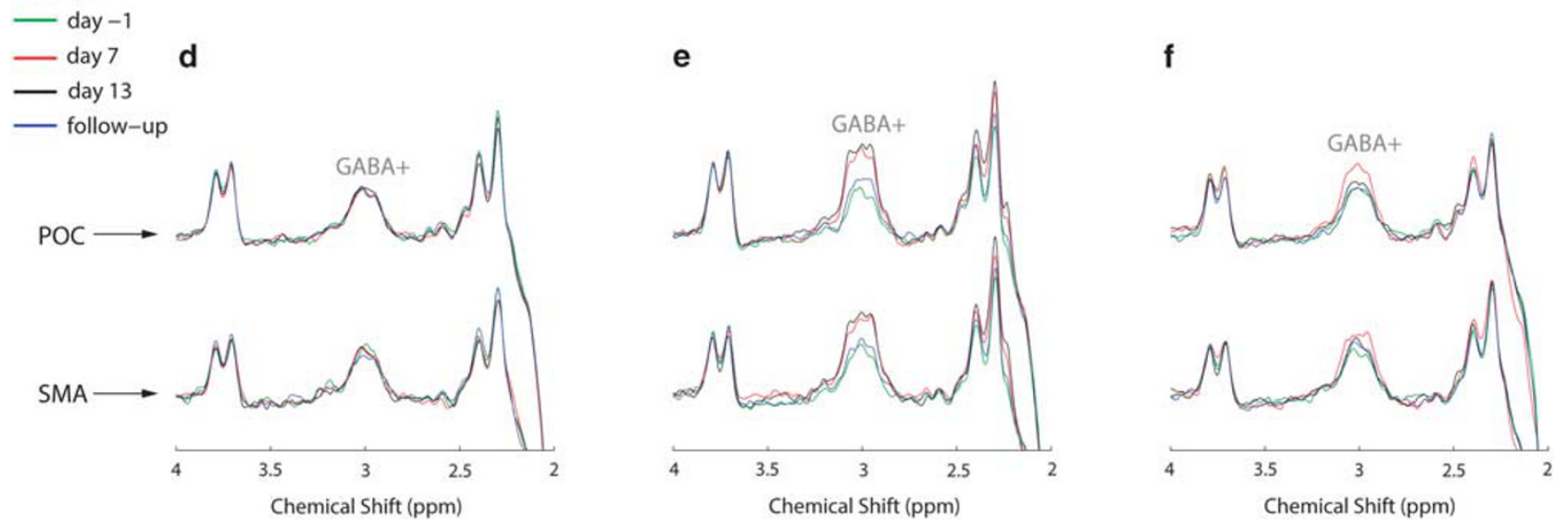

Figure 2 Sagittal and axial images ( I mm slices) from the 3D high-resolution TI-weighted MP-RAGE image data that show the typical prescription of the (a) POC and (b) SMA 'H MRS voxels (red box). (c) Baseline MEGAPRESS 'H MRS POC data recorded from subject I (CPP-I I5 cohort) and subject 2 (placebo cohort). The data are plotted using the same vertical scaling, and the spectral features and assigned signal assignments are briefly expanded upon within the main text. The bottom panel then shows a series of overlaid multiple time point POC and SMA spectra for (d) subject 2, (e) subject I, and (f) subject 6. The spectra are expanded plots of the dashed lines shown in (c). The data presented in ( $d-f$ ) were plotted using identical vertical scaling, and each color-coded spectrum can be referenced to the scan visit using the key provided.

To quantify the POC and SMA GABA+ changes induced by CPP-115 vs placebo, the effect size was calculated for both day 7 and day 13 time points. Subject data from both cohorts were included to calculate effect sizes using the relevant pooled SD.

\section{RESULTS}

Table 1 presents mean within-voxel tissue fraction data for both treatment groups together with the mean unsuppressed water LW data. The coefficient of variation (CV) values presented for each variable were obtained by first determining the $\mathrm{CV}$ for each subject, and then calculating the mean within-subject $\mathrm{CV}$ for each cohort.

Figure $2 \mathrm{c}$ shows representative day -1 MEGA-PRESS ${ }^{1} \mathrm{H}$ MRS data recorded from the POC of two subjects who went on to receive either placebo or CPP-115. The refocused GABA+ resonance is at 3.0 p.p.m., whereas additional coedited resonances have been assigned to Glx $(\mathrm{Gln}+\mathrm{Glu})$, inverted NAA (2.0 p.p.m.), and cytosolic MM (0.9 p.p.m.). The two spectra show unremarkable qualitative differences at baseline. Figures $2 \mathrm{~b}-\mathrm{d}$ show a series of overlaid POC and SMA MEGA-PRESS ${ }^{1} \mathrm{H}$ MRS data, recorded at all four scanning time points for three separate subjects. For clarity, 
an expanded spectral region is displayed (2.0-4.0 p.p.m.), and only the primary GABA+ resonance (3.0 p.p.m.) has been assigned in each of the six separate plots. For all six subjects, the quantitative POC and SMA GABA+ concentration time courses are graphically presented in Figure 3. The mean $( \pm S D)$ baseline and follow-up POC GABA+ concentrations were $0.89 \pm 0.11$ and $0.93 \pm 0.02 \mathrm{mM}$, respectively, whereas the corresponding SMA GABA+ concentrations were $0.82 \pm 0.09$ and $0.85 \pm 0.08 \mathrm{mM}$, respectively. Most individuals receiving CPP-115 showed large increases in GABA+ concentration at the day 7 and day 13 time points, whereas the 2D J-resolved ${ }^{1} \mathrm{H}$ MRS data showed no significant concentration changes for any other POC or

Table I Tissue (GM and WM) Fractions and Unsuppressed Water LW Data Calculated for Both Treatment Groups and the Two Brain Regions under Investigation

\begin{tabular}{llllllll}
\hline $\begin{array}{l}\text { Brain } \\
\text { region }\end{array}$ & Cohort & GM & $\begin{array}{c}\text { GM } \\
(\mathbf{C V})^{\mathrm{a}}\end{array}$ & WM & $\begin{array}{c}\text { WM } \\
(\mathbf{C V})^{\mathrm{a}}\end{array}$ & $\begin{array}{c}\text { LW } \\
\mathbf{( H z )}\end{array}$ & $\begin{array}{c}\text { LW } \\
(\mathbf{C V})^{\mathrm{a}}\end{array}$ \\
\hline POC & CPP-II5 & $61 \pm 6$ & $\mathrm{I} \pm 1$ & $39 \pm 6$ & $4 \pm 2$ & $6 \pm 1$ & $6 \pm 4$ \\
& Placebo & $62 \pm 5$ & $\mathrm{I} \pm 1$ & $38 \pm 5$ & $3 \pm 2$ & $6 \pm 1$ & $3 \pm 1$ \\
SMA & CPP-II5 & $58 \pm 4$ & $\mathrm{I} \pm 1$ & $42 \pm 4$ & $3 \pm 1$ & $6 \pm 1$ & $6 \pm 5$ \\
& Placebo & $57 \pm 4$ & $2 \pm 1$ & $43 \pm 4$ & $4 \pm 1$ & $6 \pm 1$ & $3 \pm 1$ \\
\hline
\end{tabular}

The mean within-subject CV also is presented for GM content, WM content, and LW.

aThe CV was expressed as $100 \times$ SD $\div$ mean.
SMA metabolite including Cre, Cho, NAA, Glu, Gln, and Ins (data not shown). For GABA+ concentration changes, the day 7 effect sizes were 7.2 and 4.1 for the POC and SMA, respectively, and the day 13 effect size was 2.1 for both brain regions. The placebo cohort showed highly stable POC and SMA GABA+ concentrations across all four ${ }^{1} \mathrm{H}$ MRS scanning time points, with mean $\mathrm{CV}$ values of $9 \%$ and $5 \%$ calculated for the POC and SMA regions, respectively.

Figure $4 \mathrm{a}$ demonstrates the effect of subtracting baseline (day - 1) data from a spectrum acquired at day 13 for individual placebo and CPP-115 data. For the POC and SMA placebo situation, the resulting spectra show negligible residual signal over the GABA (red), the Glx (black), and MM (blue) chemical shift regions. Note that some residual signal is present at $\sim 1.9$ p.p.m. in all four spectra, owing to spurious artifacts likely arising from the frequency-selective refocusing pulses required for GABA-edited MEGA-PRESS acquisitions. The POC and SMA CPP-115 data in Figure 4 show significant resonances at 3.0 and 2.3 p.p.m., corresponding to the simultaneously refocused C4 and C2 GABA + proton resonances, respectively (see molecular structure in Figure 4a). No significant residual Glx or MM signal was detected for the CPP-115 data presented in Figure 4a, in agreement with the lack of changes in Glx or MM in the 2D $J$-resolved spectra.

Figure $4 \mathrm{~b}$ shows the 6.0-9.0 p.p.m. chemical shift region of MEGA-PRESS on-resonance data recorded from the POC of a subject who had received CPP-115 for 14 days. At day 7 and day 13 , the homocarnosine histidyl resonance clearly is
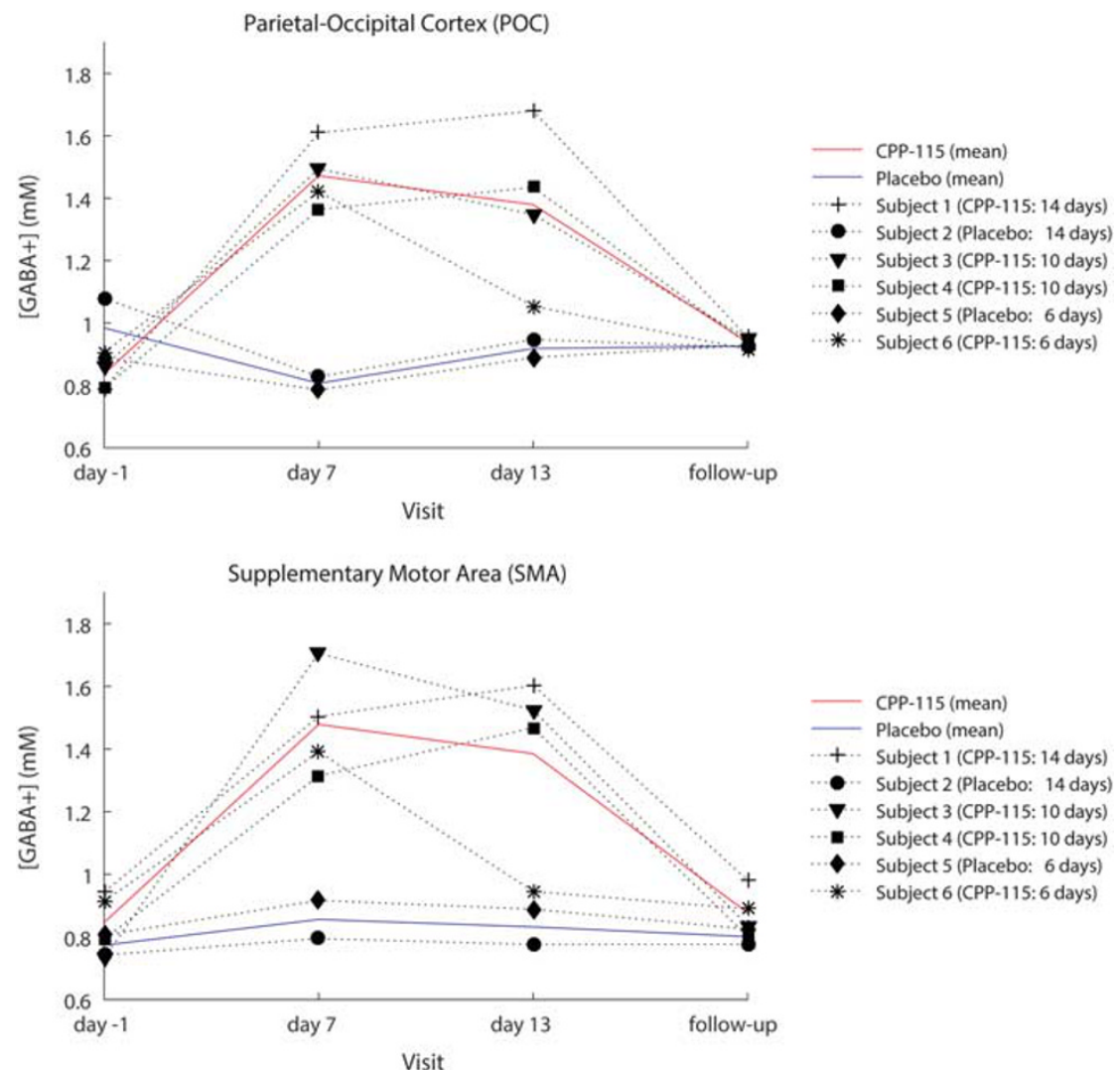

Figure 3 Time-course data for all participants showing the change in POC (top panel) and SMA (bottom panel) GABA+ concentrations across the four scan sessions. The placebo and CPP-I I5 group mean GABA+ concentration changes are also displayed for both ROls (see adjacent key). 
a

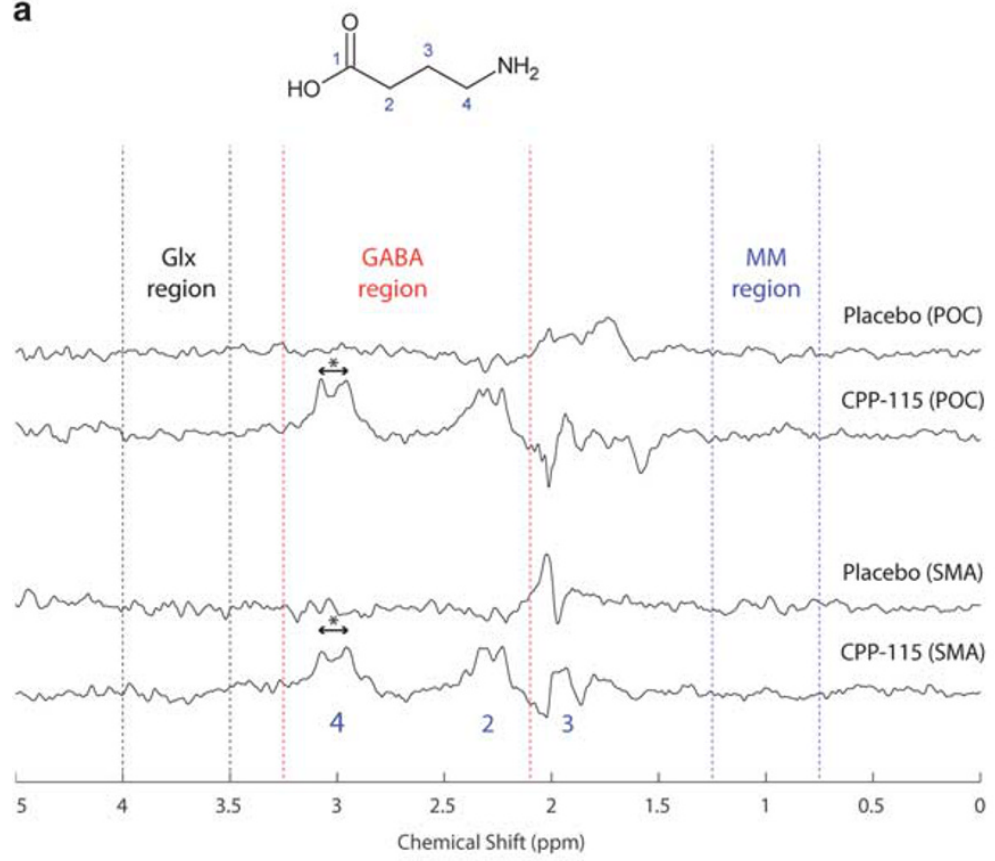

b
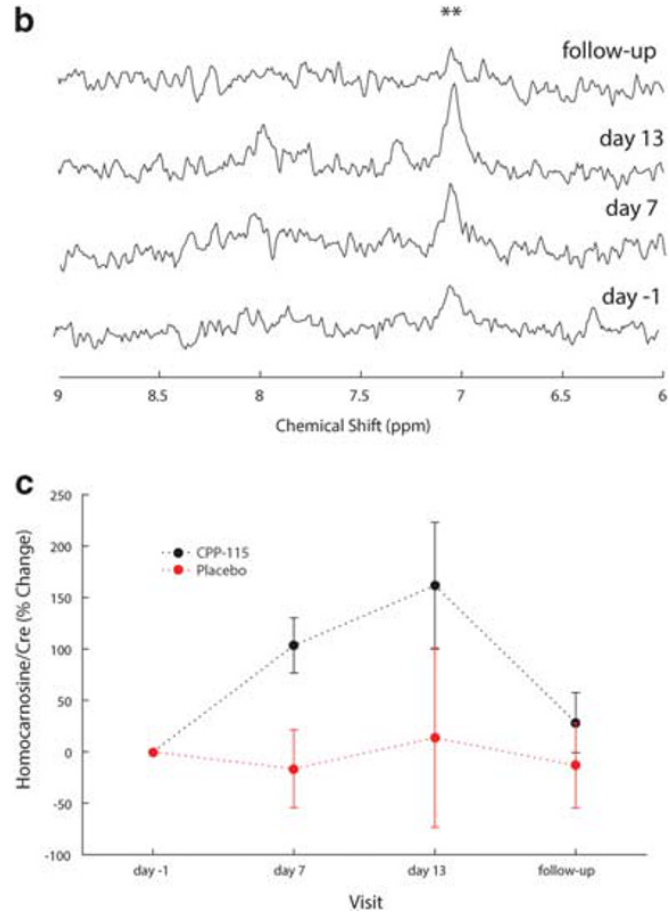

Figure 4 (a) GABA-edited 'H MRS spectra reconstructed by subtracting the baseline (day - I) data from the day 13 data in individuals receiving either placebo (subject 2) or CPP-II5 (subject I). The top two rows correspond to data reconstructed for the POC and the bottom two rows are the corresponding reconstructed SMA data (see main text for details). The same vertical scaling was used for all spectra. The peak separation highlighted by '**' is 0.128 p.p.m. ( $15.7 \mathrm{~Hz}$ ). (b) Expanded regions of MEGA-PRESS on-resonance POC 'H MRS spectra recorded at all four scanning time points from a subject who had received CPP-II 5 for 14 days (subject I). The symbol '**' denotes the homocarnosine histidyl resonance at 7.05 p.p.m. (c) Group mean time courses for POC homocarnosine for both treatment groups with y axis showing the \% change in homocarnosine/Cre compared with baseline. The error bars correspond to the calculated SE.

increased compared with day -1 , with a subsequent return to baseline levels at follow-up. For this subject, baseline and day 13 homocarnosine levels were calculated as 0.30 and $0.75 \mathrm{mM}$, respectively, whereas the GABA+ concentrations were 0.80 and $1.70 \mathrm{mM}$, respectively. Hence, CPP-115 increased both free GABA and homocarnosine by $\sim 0.45 \mathrm{mM}$, assuming the GABA+ changes are solely because of these two metabolites. Figure $4 \mathrm{c}$ shows the time courses for POC homocarnosine for both treatment groups. The CPP-115-induced SMA homocarnosine changes were of a similar magnitude with similarly stable homocarnosine levels in the placebo group (data not shown for figure clarity).

\section{DISCUSSION}

The selective and irreversible inhibition of GABA-AT is a pharmacologic mechanism that has received significant attention for the treatment of seizure disorders (Treiman, 2001). Vigabatrin remains the primary pharmacotherapy for patients with refractory seizure disorders. Because of the serious adverse side effects associated with long-term vigabatrin treatment, the development of safer nextgeneration GABA-AT inhibitors is of considerable clinical interest. CPP-115 is a novel GABA-AT inactivator that exhibits the same mechanism of action as vigabatrin yet offers several improvements including potency, specificity, and ocular safety. Preclinical data from an animal model of infantile spasms demonstrate superior efficacy over vigabatrin, with CPP-115 able to suppress spasms at doses 100 -fold lower than vigabatrin, with improved tolerance and more effective outcomes in terms of seizure reduction and duration of action (Briggs et al, 2014). CPP-115 shows favorable pharmacokinetics comparable with vigabatrin in human subjects, where it is quickly and completely orally absorbed, and rapidly eliminated. In addition, no cardiovascular or respiratory effects have been associated with CPP-115 administration (personal communication with Catalyst Pharmaceuticals). The present study employed noninvasive ${ }^{1} \mathrm{H}$ MRS technology for evaluating the pharmacodynamic characteristics of CPP-115 in human brain. The main findings and how they compare with the precedent vigabatrin MRS literature are discussed hereafter.

Collectively, the POC and SMA voxel tissue content and the water signal LW data demonstrate highly stable withinsubject measures across all MRS scanning time points. Differences in GM and WM fractions (and hence CSF content), as well as between-scan variation were negligible between the two cohorts. Hence, for this relatively shortterm chronic dosing timeframe, CPP-115 does not induce detectable macroscopic POC and SMA tissue changes, and this is important given the incidence of microvacuolation reported for other GABA-AT inhibitors (Cohen et al, 2000; John et al, 1987; Arezzo et al, 1989).

Baseline and follow-up POC and SMA GABA+ concentrations reported in the present study are in excellent agreement with previous ${ }^{1} \mathrm{H}$ MRS reports (Jensen et al, 2005). Also note the slightly higher POC GABA+ 
concentration values that likely reflects the higher GM fraction within this region of interest, and the known distribution of GABA concentration across GM and WM tissue type (Jensen et al, 2005). All subjects within the CPP-115 cohort showed robust elevations in brain GABA+ levels, with POC and SMA GABA concentrations ranging between 52 and 141\% (day 7) and 57 and 113\% (day 13) higher than baseline values. Note that Weber et al (1999) have previously demonstrated brain GABA concentration levels $60 \%$ higher than predrug values in medication-naive healthy control subjects who were administered vigabatrin at $3 \mathrm{~g}$ per day for 3 days. Considering day 7 measures, CPP-115 achieves a comparable or greater increase in GABA+ concentration with a total dose that is 16-fold lower than previous vigabatrin MRS studies. For the CPP-115 cohort, the POC and SMA GABA+ levels returned to within $2-21 \%$ baseline levels at the follow-up scan, suggesting efficient clearance of CPP-115 and the reversible resynthesis of GABA-AT. Protein resynthesis can be further appreciated when comparing the spectral plots and time-course data from subject 1 and subject 6 , participants who received CPP-115 for 14 and 6 days, respectively. For subject 1, POC and SMA GABA+ concentrations were elevated at day 7 and the levels had continued to rise at the day 13 measures. The earlier onset of GABA-AT resynthesis is clearly detected in subject 6 who showed elevated POC and SMA GABA+ concentrations at day 7 with an approach to baseline values at the day 13 measures. The level of reproducibility seen across all scanning time points in the placebo group, where $\mathrm{GABA}+$ concentration CVs ranged between 5 and $9 \%$, is well aligned with the precedent MRS literature (Near et al, 2014). Considering the large effect sizes seen for GABA+ concentration changes ( $>2$ for all day 7 and day 13 measures), future CPP-115 MRS studies can be designed to enable more rigorous statistical analysis between treatment groups.

CPP-115 exhibits excellent pharmacological target specificity with no off-target binding to 111 common pharmacological targets, including all types of GABA receptors and transporter proteins (Pan et al, 2012). With respect to the brain metabolites typically investigated using ${ }^{1} \mathrm{H}$ MRS, concentration changes in the present study were specific to GABA, suggesting that CPP-115 has negligible effect on certain measures of neuronal function (stable NAA), cell membrane synthesis/turnover (stable Cho), cerebral energetics (stable tCr), and glutamatergic metabolism (stable Gln and Glu). This contrasts with the reduced Glu and increased Gln seen with vigabatrin therapy in epileptic patients (Petroff et al, 1995) that may indicate differential metabolism in response to GABA-AT inhibitors in patients and healthy controls. Whether CPP-115 produces similar effects in a comparable epilepsy patient population remains to be established. Preclinical carbon-13 $\left({ }^{13} \mathrm{C}\right)$ MRS studies have helped shed further light on the effects of GABA-AT inhibitors. Yang and Shen (2009) reported a lower rate of Glu/Gln cycling in anesthetized rats measured from an astroglial substrate, ${ }^{13} \mathrm{C}$-labeled acetate, $24 \mathrm{~h}$ after administering a single high dose $(500 \mathrm{mg} / \mathrm{kg})$ of vigabatrin. The same report documented no change in Glu and Gln concentration. These observations likely can be explained by high extracellular GABA concentrations (increased GABAergic tone) resulting in reduced Glu release, uptake, and recycling through Gln in astrocytes. Acting through the same enzymatic mechanism of action as vigabatrin (GABA-AT inhibition), CPP-115 is likely to increase GABAergic tone and reduce Glu cycling in a similar manner, and preclinical ${ }^{13} \mathrm{C}$ MRS dynamic measures using labeled tracers are warranted to evaluate these potential effects.

Metabolite-nulled GABA-edited MEGA-PRESS data show the 0.9 p.p.m. MM signal (MM1) amplitude (and integral) to be significantly larger than the coedited 3.0 p.p.m. MM resonance (MM7) (Mescher et al, 1998), and the relative signal amplitude and integral ratios of the MM1 and MM7 peaks are constrained within a metabolite-nulled GABAedited spectrum. For the CPP-115 treatment group in the present study, subtracting baseline data from day 13 measures showed negligible POC and SMA MM1 signal, and thus the contribution of MM7 to the GABA+ changes is likely to be insignificant. This validation procedure was important as the coediting of cytosolic MM resonances is inherent to GABA J-editing-based pulse sequences, including the MEGA-PRESS ${ }^{1} \mathrm{H}$ MRS technique at clinical $\mathrm{B}_{0}$ field strengths (Harris et al, 2015; Behar et al, 1994).

At the level of MRS-detectable compounds, the change in $\mathrm{GABA}+$ concentration following CPP-115 administration appears to be exclusive to free GABA and homocarnosine. Hence, the edited 3.0 p.p.m. doublet resonance (peak separation $\sim 16 \mathrm{~Hz}$ ) that is resolved in the subtracted MEGA-PRESS data corresponds to the GABA C4 protons and the corresponding protons within the GABA moiety of homocarnosine. Because of the coresonance, additional steps were thus applied to extract quantitative homocarnosine from the MEGA-PRESS subspectra. Our estimates suggest that the magnitude of change in homocarnosine is comparable and in some cases greater than the change in free GABA. This finding is in line with studies in patient populations, where vigabatrin therapy has been shown to increase homocarnosine CSF (Menachem et al, 1988) and brain (Petroff et al, 1998) concentrations. Elevated homocarnosine levels have also been reported in patients treated with the GABAergic agents gabapentin (Petroff et al, 2000), and topiramate (Petroff et al, 1999). It is thought that homocarnosine, an inhibitory neuromodulator, serves as a storage reservoir for GABA, and is associated with improved seizure control (Henry and Theodore, 2001; Petroff et al, 1998, 2001). It should be iterated, however, that the homocarnosine changes reported for the present CPP-115 study are preliminary, owing to the suboptimal data acquisition and processing schemes employed for its quantification. Future CPP-115 spectroscopy studies will aim to more closely define GABA+ changes using MEGA-PRESS methodology in combination with additional ${ }^{1} \mathrm{H}$ MRS schemes that are optimized for homocarnosine quantification (Rothman et al, 1997).

A limitation of this work concerns the limited sample size, particularly for the placebo cohort. Immediately before the CPP-115 MRS study we performed a small test-retest reproducibility study to measure POC and SMA GABA+ levels in four healthy control subjects (two separate scans separated by a 1-week period for each subject), utilizing the same MRI scanner, identical scanner hardware, and acquisition parameters. The resulting $\mathrm{CV}$ values for the POC and SMA regions were $9 \%$ and $4 \%$, respectively, that closely 
mirrored the $\mathrm{CV}$ results from the placebo cohort in the present study, and serve to enhance confidence in the GABA + changes induced by CPP- 115 .

The data from the present study demonstrate the utility of ${ }^{1} \mathrm{H}$ MRS as a noninvasive means for measuring CPP-115 target engagement, and for monitoring GABA-AT resynthesis following drug cessation. Future ${ }^{1} \mathrm{H}$ MRS studies will be conducted to characterize and accurately delineate the acute GABA+ concentration changes associated with CPP-115 administration, and dose-descending studies will be critical for optimizing drug safety and tolerability. ${ }^{1} \mathrm{H}$ MRS studies will ultimately provide invaluable information for correlating CPP-115-induced GABA+ concentration changes with clinical response in patient populations. Given its improved efficacy and safety profile, CPP-115 could find potential therapeutic roles in a range of other domains where GABAergic deficits are well established, including substance abuse disorders (Ke et al, 2004,Prescot et al, 2013), mood disorders (Mason and Krystal, 2006), and a range of neurological disease such as Tourette's syndrome (Draper et al, 2014).

\section{FUNDING AND DISCLOSURE}

SRM and GI are employed by Catalyst Pharmaceuticals, the sponsor of the present study and developer of CPP-115. The authors declare no conflict of interest.

\section{ACKNOWLEDGMENTS}

We thank the study participants and are grateful for the logistical assistance provided by staff at PRA Health Sciences, UT. This work was entirely supported by Catalyst Pharmaceuticals, whose role was to supply study drug (CPP-115 and placebo) and to consult on any medical issues that arose during the study. PFR is supported by the National Institute on Drug Abuse (NIDA) funding award (NIDA K24 DA015116).

\section{REFERENCES}

Arezzo JC, Schroeder CE, Litwak MS, Steward DL (1989). Effects of vigabatrin on evoked potentials in dogs. Br J Clin Pharmacol 27 (Suppl 1): 53S-60S.

Behar KL, Rothman DL, Spencer DD, Petroff OA (1994). Analysis of macromolecule resonances in $1 \mathrm{H}$ NMR spectra of human brain. Magn Reson Med 32: 294-302.

Briggs SW, Mowrey W, Hall CB, Galanopoulou AS (2014). CPP-115, a vigabatrin analogue, decreases spasms in the multiple-hit rat model of infantile spasms. Epilepsia 55: 94-102.

Cohen JA, Fisher RS, Brigell MG, Peyster RG, Sze G (2000). The potential for vigabatrin-induced intramyelinic edema in humans. Epilepsia 41: 148-157.

Doumlele K, Conway E, Hedlund J, Tolete P, Devinsky O (2016). A case report on the efficacy of vigabatrin analogue (1S, 3S)-3amino-4-difluoromethylenyl-1-cyclopentanoic acid (CPP-115) in a patient with infantile spasms. Epilepsy Behav Case Rep 6: 67-69.

Draper A, Stephenson MC, Jackson GM, Pepes S, Morgan PS, Morris PG et al (2014). Increased GABA contributes to enhanced control over motor excitability in Tourette syndrome. Curr Biol 24: 2343-2347.

Edden RA, Puts NA, Harris AD, Barker PB, Evans CJ (2014). Gannet: a batch-processing tool for the quantitative analysis of gamma-aminobutyric acid-edited MR spectroscopy spectra. J Magn Reson Imaging 40: 1445-1452.

Gajcy K, Lochynski S, Librowski T (2010). A role of GABA analogues in the treatment of neurological diseases. Curr Med Chem 17: 2338-2347.

Grant SM, Heel RC (1991). Vigabatrin. A review of its pharmacodynamic and pharmacokinetic properties, and therapeutic potential in epilepsy and disorders of motor control. Drugs 41: 889-926.

Gruetter R (1993). Automatic, localized in vivo adjustment of all first- and second-order shim coils. Magn Reson Med 29: 804-811.

Harris AD, Puts NA, Barker PB, Edden RA (2015). Spectral-editing measurements of GABA in the human brain with and without macromolecule suppression. Magn Reson Med 74: 1523-1529.

Henry TR, Theodore WH (2001). Homocarnosine elevations: a cause or a sign of seizure control? Neurology 56: 698-699.

Jensen JE, Frederick Bde B, Renshaw PF (2005). Grey and white matter GABA level differences in the human brain using two-dimensional, J-resolved spectroscopic imaging. NMR Biomed 18: $570-576$.

John RA, Rimmer EM, Williams J, Cole G, Fowler LJ, Richens A (1987). Micro-vacuolation in rat brains after long term administration of GABA-transaminase inhibitors. Comparison of effects of ethanolamine-O-sulphate and vigabatrin. Biochem Pharmacol 36: 1467-1473.

Jung MJ, Lippert B, Metcalf BW, Bohlen P, Schechter PJ (1977). gamma-Vinyl GABA (4-amino-hex-5-enoic acid), a new selective irreversible inhibitor of GABA-T: effects on brain GABA metabolism in mice. J Neurochem 29: 797-802.

Ke Y, Streeter CC, Nassar LE, Sarid-Segal O, Hennen J, YurgelunTodd DA et al (2004). Frontal lobe GABA levels in cocaine dependence: a two-dimensional, J-resolved magnetic resonance spectroscopy study. Psychiatry Res 130: 283-293.

Mason GF, Krystal JH (2006). MR spectroscopy: its potential role for drug development for the treatment of psychiatric diseases. NMR Biomed 19: 690-701.

Mattson RH, Petroff O, Rothman D, Behar K (1994). Vigabatrin: effects on human brain GABA levels by nuclear magnetic resonance spectroscopy. Epilepsia 35(Suppl 5): S29-S32.

Menachem EB, Persson LI, Schechter PJ, Haegele KD, Huebert N, Hardenberg J et al (1988). Effects of single doses of vigabatrin on CSF concentrations of GABA, homocarnosine, homovanillic acid and 5-hydroxyindoleacetic acid in patients with complex partial epilepsy. Epilepsy Res 2: 96-101.

Mescher M, Merkle H, Kirsch J, Garwood M, Gruetter R (1998). Simultaneous in vivo spectral editing and water suppression. NMR Biomed 11: 266-272.

Near J, Ho YC, Sandberg K, Kumaragamage C, Blicher JU (2014). Long-term reproducibility of GABA magnetic resonance spectroscopy. Neuroimage 99: 191-196.

Pan Y, Gerasimov MR, Kvist T, Wellendorph P, Madsen KK, Pera E et al (2012). (1S, 3S)-3-amino-4-difluoromethylenyl-1-cyclopentanoic acid (CPP-115), a potent gamma-aminobutyric acid aminotransferase inactivator for the treatment of cocaine addiction. J Med Chem 55: 357-366.

Perucca P, Gilliam FG (2012). Adverse effects of antiepileptic drugs. Lancet Neurol 11: 792-802.

Petroff OA, Rothman DL, Behar KL, Mattson RH (1995). Initial observations on effect of vigabatrin on in vivo $1 \mathrm{H}$ spectroscopic measurements of gamma-aminobutyric acid, glutamate, and glutamine in human brain. Epilepsia 36: 457-464.

Petroff OA, Rothman DL, Behar KL, Mattson RH (1996). Low brain GABA level is associated with poor seizure control. Ann Neurol 40: 908-911.

Petroff OA, Mattson RH, Behar KL, Hyder F, Rothman DL (1998). Vigabatrin increases human brain homocarnosine and improves seizure control. Ann Neurol 44: 948-952. 
Petroff OA, Hyder F, Mattson RH, Rothman DL (1999). Topiramate increases brain GABA, homocarnosine, and pyrrolidinone in patients with epilepsy. Neurology 52: 473-478.

Petroff OA, Hyder F, Rothman DL, Mattson RH (2000). Effects of gabapentin on brain GABA, homocarnosine, and pyrrolidinone in epilepsy patients. Epilepsia 41: 675-680.

Petroff OA, Hyder F, Rothman DL, Mattson RH (2001). Homocarnosine and seizure control in juvenile myoclonic epilepsy and complex partial seizures. Neurology 56: 709-715.

Prescot AP, Renshaw PF (2013). Two-dimensional J-resolved proton MR spectroscopy and prior knowledge fitting (ProFit) in the frontal and parietal lobes of healthy volunteers: assessment of metabolite discrimination and general reproducibility. J Magn Reson Imaging 37: 642-651.

Prescot AP, Renshaw PF, Yurgelun-Todd DA (2013). gammaAmino butyric acid and glutamate abnormalities in adolescent chronic marijuana smokers. Drug Alcohol Depend 129: 232-239.

Rothman DL, Behar KL, Prichard JW, Petroff OA (1997). Homocarnosine and the measurement of neuronal $\mathrm{pH}$ in patients with epilepsy. Magn Reson Med 38: 924-929.

Schulte RF, Boesiger P (2006). ProFit: two-dimensional priorknowledge fitting of J-resolved spectra. NMR Biomed 19: 255-263.
Schulte RF, Lange T, Beck J, Meier D, Boesiger P (2006). Improved two-dimensional J-resolved spectroscopy. NMR Biomed 19: 264-270.

Smith SM (2002). Fast robust automated brain extraction. Hum Brain Mapp 17: 143-155.

Smith SM, Jenkinson M, Woolrich MW, Beckmann CF, Behrens TE, Johansen-Berg $\mathrm{H}$ et al (2004). Advances in functional and structural MR image analysis and implementation as FSL. Neuroimage 23(Suppl 1): S208-S219.

Treiman DM (2001). GABAergic mechanisms in epilepsy. Epilepsia 42(Suppl 3): 8-12.

Weber OM, Verhagen A, Duc CO, Meier D, Leenders KL, Boesiger P (1999). Effects of vigabatrin intake on brain GABA activity as monitored by spectrally edited magnetic resonance spectroscopy and positron emission tomography. Magn Reson Imaging 17: 417-425.

Yang J, Shen J (2009). Elevated endogenous GABA concentration attenuates glutamate-glutamine cycling between neurons and astroglia. J Neural Transm (Vienna) 116: 291-300.

Yogeeswari P, Ragavendran JV, Sriram D (2006). An update on GABA analogs for CNS drug discovery. Recent Pat CNS Drug Discov 1: 113-118.

Zhang Y, Brady M, Smith S (2001). Segmentation of brain MR images through a hidden Markov random field model and the expectationmaximization algorithm. IEEE Trans Med Imaging 20: 45-57. 